\title{
A Comparative Study of Computerized Memory Test and The Korean version of the Consortium to Establish a Registry for Alzheimer's Disease Assessment Packet for Assessing Memory Function in the Elderly
}

\author{
Min-Sup Shin ${ }^{1}$, Jayun $\mathrm{Choi}^{2}$, Ryu-Yeon Ahn², Dong-Young Lee', and Jun-Soo Kwon ${ }^{1 凶}$ \\ ${ }^{1}$ Department of Psychiatry, College of Medicine, Seoul National University, Seoul, Republic of Korea \\ ${ }^{2}$ Department of Psychiatry, Seoul National University Hospital, Seoul, Republic of Korea
}

Objective The aim of this study was to examine the concurrent validity of a newly developed computerized memory diagnostic system (MDS) with the Korean version of the Consortium to Establish a Registry for Alzheimer's Disease Assessment Packet (CERAD-K).

Methods Subtests of the MDS and CERAD-K, including the auditory-verbal, visuo-spatial, and working memory tests, were administered to 43 adults aged 60 to 74 years. We calculated the correlations between the subtest scores of the MDS and CERAD-K to examine the concurrent validity of the MDS.

Results We found significant correlations between the subtest scores in the verbal-auditory memory, including immediate recall, delayed recall, and delayed recognition. The working memory subtest scores between the MDS and CERAD-K also showed a significant positive correlation.

Conclusion We verified the concurrent validity of the memory subtests in the MDS for the elderly. The results of the present study suggest that the MDS could be a valuable tool for an efficient and valid assessment of memory function.

Psychiatry Investig 2016;13(6):590-594

Key Words Memory, Neuropsychological test, Validity.

\section{INTRODUCTION}

In recent years, the risk of developing mild cognitive impairment (MCI) and dementia in normal aging adults has dramatically increased in Korea. The proportion of adults who are 65 and older in the Korean society has increased to $12.7 \%$ of the total population in 2014 . This is expected to increase to $20 \%$ by 2026 . The elderly population at high risk of developing dementia is expected to account for $24.3 \%$ and $40.1 \%$ of the total population in 2030 and 2060 , respectively. This will significantly increase their socioeconomic burden; therefore, there is an urgent need for prevention of dementia

Received: January 28, 2015 Revised: July 1, 2015

Accepted: July 19, 2015 Available online: November 2, 2016

$\triangle$ Correspondence: Min-Sup Shin, $\mathrm{PhD}$

Department of Psychiatry, Seoul National University College of Medicine, 101 Daehak-ro, Jongno-gu, Seoul 03080, Republic of Korea

Tel: +82-2-2072-2454, Fax: +82-2-747-9063, E-mail: shinms@snu.ac.kr

(c) This is an Open Access article distributed under the terms of the Creative Commons Attribution Non-Commercial License (http://creativecommons.org/licenses/by$\mathrm{nc} / 3.0$ ) which permits unrestricted non-commercial use, distribution, and reproduction in any medium, provided the original work is properly cited. and early intervention.

The symptoms of dementia begin with amnesia, followed by a decrease in cognitive functions, such as language, sense of direction, and computational power. In addition, dementia patients can develop mood disorders, such as depression with changing personality and emotional behaviors. These factors aggravate the burden on caregivers, and affect the patients' overall quality of life and social environment. Therefore, early identification of the initial symptoms of cognitive decline is important to enable a therapeutic intervention for the prevention of dementia. Subjective memory complaints in the elderly may not reliably reflect real memory impairments based on a lack of information and insight into their own memory function; therefore, regular verification of memory function through accurate and efficient examination is crucial for the successful prevention of dementia and rehabilitation.

A decline in memory function, particularly in learning and memorizing of verbal and non-verbal content material, such as words, numbers, and pictures that are closely related to 
daily life, can occur in patients with dementia, brain injuries, and psychiatric illnesses, such as depression and anxiety. Standardized tests, with verified reliability and validity, are required to accurately evaluate cognitive functions. Traditional paper-and-pencil tests are advantageous because their reliability and validity has been confirmed in both research and clinical settings; however, it is difficult to control situational factors in these tests, such as differences between examiners, and the interpretation of the results is time-consuming. ${ }^{1-5}$

The screening and detection of $\mathrm{MCI}$ in an annual comprehensive health check, including memory tests and a physical examination, in middle-aged adults might prevent the risk and onset of dementia. Early detection and screening of cognitive functions might slow the increase in dementia that is occurring with the increasing proportion of the elderly in the population. However, the one-on-one paper-and-pencil tests that are currently used are difficult to carry out in a large-scale for the general population due to time and cost limitations.

A standardized computerized test is advantageous because it can be administered to a large number of people and the results can be viewed immediately using an automatic scoring system. Moreover, it can record speed and accuracy more precisely and has higher efficiencies with materials and time. ${ }^{6}$ Tools such as the Cambridge Neuropsychological Test Automated Battery (CANTAB) are computerized; however, this was developed to measure the overall cognitive function rather than focus on evaluating memory. In addition, majority of these tests take a long time to administer and have not been standardized in a Korean population; therefore, they are difficult to implement in clinical practice in Korea.

Shin and Kwon ${ }^{7}$ have developed a computerized memory test called the Memory Diagnostic System (MDS). They conducted a standardization study to calculate the age norms for this test and examined its reliability and validity in 640 normal Korean adults aged 40 to 74 years. This computerized memory test could be useful in clinics and health centers because it can be administered with simple instructions without the need for a specialized clinician. However, the concurrent validity of the MDS has not been verified with the paperand-pencil test that is widely used in the clinic. The purpose of this study was to examine the concurrent validity of the MDS with the Korean Version of the Consortium to Establish a Registry for Alzheimer's Disease Assessment Packet (CERAD$\mathrm{K})$, which is a widely used formal diagnostic tool for dementia. ${ }^{8}$

\section{METHODS}

\section{Participants}

Forty-three adults aged 60-74 years who had a subjective memory complaint were recruited for this study from the normal population and from the geriatric psychiatric ward of Seoul National University Hospital for dementia examination. All participants were provided with information about the purpose of the study. Participants were classified into five age groups (60-62, 63-65, 66-68, 69-71, and 72-74 years) to apply age norms of the MDS for each group. The elderly were divided into groups of 3 years unlike the middle-aged subjects, who were divided into groups of 5 years, because memory decline is more precipitous after the age of 60 years. ${ }^{9}$ Exclusion criteria included alcohol/substance dependence, medical conditions that damage cognitive function, psychiatric symptoms, and illiteracy. We included all subjects who had subjective memory complaint and did not meet the exclusion criteria. Since the purpose of this study was to confirm concurrent validity of MDS with paper and pencil based test rather than screening of MCI, we did not discriminate between MCI and healthy subjects.

All subjects signed an Institutional Review Board (IRB)approved, written consent form (IRB approval number C-1408059-603).

\section{Measures}

\section{The Memory Diagnostic System}

The MDS is a computerized test battery that comprehensively assesses cognitive abilities, such as verbal, visuo-spatial, attention, working memory, and executive function. The MDS is composed of two versions: for middle aged (40-59 years) and for elderly (60-74 years) adults. In this study, the auditory-verbal, visuo-spatial, and working memory subtests were conducted and compared with the CERAD-K scores.

In the auditory-verbal memory subtest, a word list (15 and 12 words for middle aged and elderly adults, respectively) was presented through an auditory channel at a rate of one word per second. Participants were required to immediately recall the word list. This was repeated three times. Fifteen minutes later, a delayed recall was carried out without forewarning the participants. Following this, several words were presented visually and participants were asked to recognize the target words among interference words, which had not been previously presented.

The visuo-spatial memory subtest was conducted by presenting six figures in a $2 \times 3$ grid for 10 seconds. The participants were required to remember the shape and place of the figures, and then select the target figures from 30 examples. This was repeated three times. Stimuli were created by transforming the Korean letters to differentiate them from other visual memory tests. Both immediate and delayed recognition was carried out as described for the auditory-verbal memory subtest.

During a 15-min interval, working memory subtests were 
conducted. A letter-number sequence was used to assess verbal-auditory working memory. Participants were asked to rearrange the mixed Korean letters and numbers based on a given rule. The inter-stimulus interval was $1000 \mathrm{~ms}$. In the visuo-spatial working memory subtest, nine bulbs were presented in a $3 \times 3$ grid on a computer screen. Each bulb lit up for $500 \mathrm{~ms}$ at $1000 \mathrm{~ms}$ intervals in a given order. Participants were asked to remember the sequence of lighted bulbs and touch the bulbs in the same order. Backward trials were conducted right after the forward trials were completed.

\section{The Korean version of the Consortium to Establish} a Registry for Alzheimer's Disease Assessment Packet

The CERAD-K is a paper and pencil-based memory test battery developed in Korea. Its reliability and validity have been verified and it is widely used in clinical practice. In this study, we administered subtests of the CERAD-K: word list memory, word list delayed recall and recognition, and digit span, to confirm the concurrent validity of the MDS. A word list memory is a free recall memory test that assesses the learning ability for new verbal information. A list of 10 items was presented. Three trials were performed and participants were asked to speak each word aloud, and then recall as many items as possible. Fifteen minutes later, delayed recall and recognition tests were administered. To evaluate the visuo-spatial memory, we used a constructional recall test because the CERAD$\mathrm{K}$ does not include a subtest that assesses visuo-spatial memory directly. Participants were asked to copy four figures, and then redraw the figures from memory after a few minutes. Forward and backward digit span tests were administered to evaluate the working memory.

\section{Procedure}

We used the MDS to assess memory function. We compared only memory and working memory functions because CERAD-K does not include other areas such as sustained attention. Participants signed a consent form prior to the study and were notified that they could opt out of the study at any point. The MDS was administered in a quiet room by a clinical psychology trainee using a computerized program, without the involvement of the researchers. The clinical trainee was present in case the participants complained of tiredness or there were unexpected problems. There was a 1-hour interval between administration of the MDS and CERAD-K to prevent any interference effect.

\section{Analysis}

We performed the statistical analyses using PASW 18.0. The MDS automatically calculated a standardized score for each age group using the mean and standard deviation. We transformed the raw CERAD-K scores to standardized scores for each age group using the same method. Any gender differences were assessed by t-test. Pearson's correlation analyses were performed to verify the concurrent validity between the MDS and CERAD-K.

\section{RESULTS}

\section{Demographic characteristics and sex differences}

Patients who were in the hospital for a memory function evaluation and non-patients who complained of subjective memory decline were recruited to participate in this study. Forty-three participants (men: 14, women: 29) with a mean age of 67.49 years $(\mathrm{SD}=4.26)$, completed both the MDS and CERAD-K. Mean MMSE-K score was 25.79 (SD=3.54) and education years were 11.35 ( $\mathrm{SD}=3.72)$.

There was no significant difference between genders in the word list memory, word list delayed recall and recognition, constructional recall, and digit span, as measured by the CERAD$\mathrm{K}$. There were no significant differences between gender in the auditory-verbal memory and visuo-spatial memory, as measured by the MDS; however, men showed a significantly higher working memory score than women (Table 1).

\section{Correlation analysis between subtest scores}

\section{Auditory-verbal memory}

There was a significant positive correlation between the auditory-verbal immediate recall and word list immediate recall subtest scores of the MDS and CERAD-K, respectively ( $\mathrm{r}=$ $0.64, \mathrm{p}<0.001)$. In addition, there was a significant correlation between the auditory-verbal delayed recall and word list delayed recall scores $(\mathrm{r}=0.73, \mathrm{p}<0.001)$ (Table 2$)$. There was a significant positive correlation between the scores in the delayed recognition tests of the MDS and CERAD-K ( $\mathrm{r}=0.56$, $\mathrm{p}<0.001$ ) (Table 2).

\section{Visual-spatial memory}

The MDS visuo-spatial delayed recognition subtest score and the CERAD-K constructional delayed recall score was not significantly correlated $(\mathrm{r}=0.28)$ (Table 2 ).

\section{Working memory}

The MDS working memory scores showed a significant positive correlation with the CERAD-K digit span scores $(r=$ $0.47, \mathrm{p}<0.001$ ) (Table 2).

\section{DISCUSSION}

It is well known that there are age-related changes in the 
Table 1. Descriptive statistics and gender difference of MDS and CERAD-K

\begin{tabular}{|c|c|c|c|c|}
\hline & Total $(\mathrm{N}=43)$ & Male $(\mathrm{N}=14)$ & Female $(\mathrm{N}=29)$ & $\mathrm{t}$ \\
\hline \multicolumn{5}{|l|}{ CERAD-K } \\
\hline Word list immediate recall & $10.10(3.82)$ & $10.16(3.82)$ & $10.06(3.97)$ & -0.81 \\
\hline Word list delayed recall & $8.85(3.45)$ & $8.49(4.37)$ & $9.03(3.00)$ & 0.47 \\
\hline Wordlist recognition & $9.61(3.12)$ & $9.92(3.47)$ & $9.47(3.00)$ & -0.43 \\
\hline Visual recognition & $8.87(3.58)$ & $9.02(3.86)$ & $8.79(3.51)$ & -0.19 \\
\hline Digit span backward & $4.79(2.12)$ & $5.36(2.71)$ & $4.52(1.77)$ & -1.22 \\
\hline \multicolumn{5}{|l|}{ MDS } \\
\hline Word list immediate recall & $9.50(3.33)$ & $8.14(4.01)$ & $10.17(2.76)$ & 1.92 \\
\hline Word list delayed recall & $8.13(3.65)$ & $7.07(4.09)$ & $8.64(3.38)$ & 1.33 \\
\hline Wordlist recognition & $8.43(4.12)$ & $7.66(4.93)$ & $8.80(3.71)$ & 0.84 \\
\hline Visual recognition & $9.60(3.34)$ & $9.73(3.88)$ & $9.53(3.21)$ & -0.18 \\
\hline Working memory & $9.63(2.92)$ & $10.94(3.85)$ & $9.00(2.26)$ & $-2.08^{*}$ \\
\hline
\end{tabular}

Table 2. Correlation between MDS and CERAD-K

\begin{tabular}{|c|c|c|c|c|c|}
\hline \multirow[b]{2}{*}{ CERAD-K } & \multicolumn{5}{|c|}{ MDS } \\
\hline & $\begin{array}{c}\text { Word list } \\
\text { immediate recall }\end{array}$ & $\begin{array}{c}\text { Word list } \\
\text { delayed recall }\end{array}$ & $\begin{array}{l}\text { Word list } \\
\text { recognition }\end{array}$ & $\begin{array}{c}\text { Visual } \\
\text { recognition }\end{array}$ & $\begin{array}{l}\text { Working } \\
\text { memory }\end{array}$ \\
\hline Word list immediate recall & $0.64^{* * *}$ & & & & \\
\hline Word list delayed recall & & $0.73^{* * *}$ & & & \\
\hline Word list recognition & & & $0.56^{* * *}$ & & \\
\hline Visual recognition & & & & 0.28 & \\
\hline Digit span backward & & & & & $0.47^{* * *}$ \\
\hline
\end{tabular}

${ }^{* * *} \mathrm{p}<0.001$. CERAD-K: Consortium to Establish a Registry for Alzheimer's Disease Assessment Packet, MDS: memory diagnostic system

various types of cognitive function. Although previously acquired information and skills do not appear to decline sharply with age, memory for new information and other abilities, such as sensory integration and information processing speed, show age-related decline. ${ }^{10}$ In addition, recovery from premorbid onset of dementia is difficult; therefore, early detection of patients with MCI, which is the clinical stage that precedes dementia, is crucial in the prevention of dementia. ${ }^{11}$ Thus, an efficient, reliable, and cost-effective computerized memory test would be a useful tool for the early detection of MCI and to assess the risk of dementia in the general population.

The purpose of this study was to confirm whether the MDS, a newly developed computerized test for Korean elderly adults, was as effective at evaluating memory function as the existing CERAD-K. We analyzed the correlation between subtest scores in the MDS and CERAD-K, which is a paper and pencil-based test that has been verified for reliability and validity and is widely used in the clinic. To summarize our findings, we found no significant gender differences in immediate recall, delayed recall, and delayed recognition of auditory-verbal or visuo-spatial memory. However, men showed significantly higher working memory scores than women in the MDS.

There was a significant positive correlation between the MDS and CERAD-K auditory-verbal memory subtest scores. Immediate and delayed recall, and delayed recognition showed a significant relationship; therefore, we have confirmed that the MDS has concurrent validity with the CERAD-K, regardless of the method of administration. In addition, we found a moderately positive correlation between the digit span scores in the CERAD-K and the working memory test scores in the MDS. Working memory is related to the frontal lobe activation and shows clear functional decline with aging because it requires more mental effort and cognitive resources. ${ }^{12}$ Therefore, we expect that the working memory subtest of the MDS is a simple and accurate assessment of the working memory that could be used to assess functional decline. Although there was a gender difference in working memory in the MDS, it was not significant in the CERAD-K. Robert and Savoie $^{13}$ also reported that there was no gender difference in working memory resource. In the way that working memory is closely related to cognitive strategy, other factors such as education level could be related to gender difference, and men showed significantly higher education level than wom- 
en $(t=-2.75, p<0.05)$. There was no significant correlation between the MDS and CERAD-K visuo-spatial memory subtest scores. Constructional recall in the CERAD-K requires visual constructional ability; therefore, this could interrupt the accurate assessment of visuo-spatial memory using the CERAD-K. The visuo-spatial memory subtest of the MDS requires participants to touch a computer screen, whereas the constructional recall test in the CERAD-K requires participants to copy figures; therefore, other factors, such as visual motor coordination may affect the correlation efficiency between the two tests. Taken together, the results of this study showed that the auditory-verbal and working memory subtests of the MDS were a valid assessment when compared with the paper and pencil-based CERAD-K test.

There are some limitations to this study. First, the sample size and age range (60-74) are limited; therefore, it is hard to generalize these results to a wider population. Second, participants were patients who were suspected of a real cognitive impairment, and non-patients who had complained of subjective memory decline, which could reduce the homogeneity of the sample. We assessed the subjective memory compliant; however, there was no objective examination of memory function. Thus, it is possible that these two groups have different characteristics. Third, it was hard to recruit suitable subjects because of the limited age range and time, and the sample size was small enough to not meet the significance level. In spite of these limitations, the significant correlation between a newly developed computerized memory test and the paper and pencil based test that has been verified for reliability and validity is a meaningful result. Although many Korean elderly people are not familiar with computer, the MDS is carried out with simple button and touch screen without any complex operation. Moreover, unlike the paper and pencil test, the MDS could be conducted without professional clinician and presents automated interpretation immediately after the test.

There are a few computerized cognitive tests, which already have been rapidly adopted in clinical fields. Computer-Administered Neuropsychological Screen for Mild Cognitive Impairment (CANS-MCI) is a tool for early detection MCI and includes test of language, memory, and executive function. ${ }^{14}$ However, it does not assess attention, which is core ability for encoding information. Computerized Neuropsychological Test Battery (CNTB) includes wider areas such as motor speed, information processing, attention, memory, language, and spatial abilities, but this battery is fully operated by a clinician. ${ }^{15}$ The MDS covers these shortcomings, and therefore, we believe that the MDS could be widely used in clinical settings and in studies to accurately and efficiently assess memory function in the normal elderly population, which has a high risk of dementia and other cognitive impairments. In addition, using the MDS to annually screen memory function would identify people at an early stage of dementia and minimize functional deterioration by providing early intervention.

\section{Acknowledgments}

Supported by grant no. 04-2013-0420 from SNUH Research Fund.

\section{REFERENCES}

1. Wilson SL, McMillan TM. Computer-Based Assessment in Neuropsychology. In: Crawford JR, Parker DM, Editor. A Handbook of Neuropsychological Assessment. Lawrence Erlbaum Associates Ltd; 1992. p.413-420.

2. Gur RC, Ragland JD, Moberg PJ, Bilker WB, Kohler C, Siegel SJ, Gur RE. Computerized neurocognitive scanning: II. The profile of schizophrenia. Neuropsychopharmacology 2001;25:777-788.

3. Ballard JC. Computerized assessment of sustained attention: a review of factors affecting vigilance performance. J Clin Exep Neuropsychol 1996;18:843-863.

4. Ferris SH, Flicker C, Reisberg B. NYU computerized test battery for assessing cognition in aging and dementia. Psychopharmacol Bull 1988; 24:699-702.

5. Rhee HS. A review of the evaluation tools for cognitive perceptual performance ability. Soc Occup Ther Aged Dement 2010;4:35-46.

6. Wild K, Howieson D, Webbe F, Seelye A, Kaye J. Status of computerized cognitive testing in aging: a systematic review. Alzheimers Dement 2008;4:428-437.

7. Shin MS, Kwon JS. Memory Diagnostic System (MDS). Seoul: Brain Medic Co., Ltd; 2014.

8. Lee JH, Lee KU, Lee DY, Kim KW, Jhoo JH, Kim JH, et al. Development of the Korean Version of the Consortium to Establish a Registry for Alzheimer's Disease Assessment Packet (CERAD-K) clinical and neuropsychological assessment batteries. J Gerontol B Psychol Sci Soc Sci 2002;57:P47-P53.

9. Brickman AM, Stern Y. Aging and memory in humans. Enc Neurosci 2009;1:175-180.

10. Cheon JS. Neurocognitive assessment of geriatric patients. J Korean Soc Biol Therap Psychiatry 2000;6:126-139.

11. Lee SA, Lee WH, Song JY, Paik JW. Association between subjective memory complaints and objective cognitive functions in elderly. Korean Clin Psychol Assoc 2011;30:247-261.

12. Ackerman PL, Rolfhus EL. The locus of adult intelligence: knowledge, abilities, and nonability traits. Psychol Aging 1999;14:314-330.

13. Robert M, Savoie N. Are there gender differences in verbal and visuospatial working-memory resources? Eur J Cogn Psychol 2006;18:378397.

14. Tornatore JB, Hill E, Laboff JA, McGann ME. Self-administered screening for mild cognitive impairment: initial validation of a computerized test battery. J Neuropsychiatry Clin Neurosci 2005;17:98-105.

15. Veroff AE, Cutler NR, Sramek JJ, Prior PL, Mickelson W, Hartman JK. A new assessment tool for neuropsychopharmacologic research: the Computerized Neuropsychological Test Battery. J Geriatr Psychiatry Neurol 1991;4:211-217. 